known to the specialist palliative care team or who died during a six-month period were included.

Results Five patients were identified.

- The age of the patients ranged from 37 to 66 years (mean 53.8 years)

- Four of the patients had metastatic malignancy; one patient had a metabolic disorder

- The patients were referred with predominantly physical symptoms including pain, breathlessness and fatigue

- Social isolation and a need for advance care planning were identified once the patients were reviewed

- In four of the patients there was evidence of joint working by the two specialist teams.

Discussion We have recognised that there is a need to improve access to our services for patients with learning disabilities and have developed formal links with the learning disability team. Communication and access for staff and patients have been improved through joint working to develop a better understanding of each other's roles and a new patient information leaflet about palliative care developed with user involvement. Palliative care involvement highlighted unrecognised need, helped ensure patients' wishes for future care were elicited and that they were more involved in decisionmaking.

\section{P-54 DEVELOPING ACCESSIBLE AND INCLUSIVE PALLIATIVE CARE FOR LGBTQI PATIENTS AND THEIR CARERS}

Rebecca Lennon, Aruna Hodgson, Jo Carby, Kate Hadfield, Alison Govan, Kerry Beaumont, Sam Shale, Carol Close. Wigan and Leigh Hospice, Wigan, UK

\subsection{6/bmjspcare-2017-hospice.81}

Background The Care Quality Commission's report 'A different ending' (2016) highlighted the health inequalities that LGBTQI (lesbian, gay, bisexual, transgender, questioning and intersex) patients, and their carers, experience with end of life care. The hospice values accessibility, so a group was set up to determine whether our services are accessible and inclusive to LGBTQI patients and their carers.

$\operatorname{Aim}(\mathbf{s})$ To understand the issues facing LGBTQI patients, and their carers, at the end of life. To recognise their experience of using the hospice's services and explore service development ideas with staff and external stakeholders.

Methods A multidisciplinary working group was set up, which included medical and nursing staff, the communications manager, volunteer chaplain and external representation from the local LGBTQI community. The group used a service audit tool (LGBT Age 2015) to discuss the current services and generate ideas. A literature review was conducted to understand LGBTQI issues at the end of life and a staff survey performed to understand their knowledge and attitudes about LGBTQI issues. Expert advice was sought from Marie Curie, Macmillan, other hospices and a local LGBTQI healthcare professional group.

Results The hospice will increase its visibility to the LGBTQI population and service users by promoting the project through local and social media, attending the local Pride festival and displaying the synonymous rainbow flag in reception. Hospice policies, monitoring form and leaflets have been reviewed and will be made public, which include clear confidentiality and anti-discriminatory statements. Education sessions for staff will be designed and members of the working group will become LGBTQI champions.

Conclusions By increasing the hospice's visibility to the LGBTQI community, understanding their needs and making it clear that we are an accessible and safe service to use, our LGBTQI patients, and their carers, will have a better end of life care experience at the hospice.

\section{P-55 A STRATEGY TO ADDRESS INEQUALITY IN PALLIATIVE CARE}

Karen Chumbley. St Helena Hospice, Colchester, UK

\subsection{6/bmjspcare-2017-hospice.82}

Background Palliative care is inequitably distributed in our society according to diagnosis, age, postcode, wealth and social group (Sleeman, 2016; Dixon \& King, 2015; Gomes et al., 2006; Care Quality Commission, 2016; Gao et al., 2013).

Aim Hospice strategic development addressing local inequality in palliative care provision.

Method We evaluated the equality of local service provision in relation to postcode, diagnosis and deprivation indices. We also examined the access equity to the local electronic palliative care coordination system (EPaCCs). We identified an inequality in access to hospice services, particularly inpatient beds, dependant on postcode. Only $3 \%$ of people who died from the coastal area died in the hospice, compared to $6 \%$ who live locally. The majority of those accessing hospice services had a diagnosis of cancer. Numbers of people accessing hospice services with a primary diagnosis of dementia were low. Only $1 \%$ of people on the local electronic palliative care register lived in a care home despite $25 \%$ of deaths occurring there.

The hospice developed an action plan to address these inequalities:

- Partnership working with the local mental health team to improve palliative care for those with dementia

- GP Associate roles to promote timely identification of those approaching the end of life.

- Increased multidisciplinary work with renal, respiratory, heart failure and neurology teams.

- A joint project with Macmillan to outreach to marginalised groups.

- Care home education to promote access to EPaCCs.

- A business plan for hospice beds in the coastal area is under development.

Results Over the last two years the number of people with a primary diagnosis of dementia or frailty accessing hospice care has quadrupled, the proportion of hospice patients with noncancer diagnoses has increased from $21 \%$ to $33 \%$ and the proportion of people on the EPaCCs system living in a care home has increased from $1 \%$ to $12 \%$.

Conclusion Hospices strategy can address the inequalities in palliative care provision. 